\section{GENETIC DISRUPTION OF NEGATIVE IMMUNE REGULATORS TO ENHANCE CAR-T EFFICACY AGAINST SOLID TUMORS}

David Mai ${ }^{*}$, Omar Johnson, Carl June. University of Pennsylvania, Philadelphia, PA, USA

Background CAR-T cell therapy has demonstrated remarkable success in hematological malignancies but displays limited efficacy in solid tumors, which comprise most cancer cases. Recent studies suggest that CAR-T cell failure via $T$ cell exhaustion is characterized by decreased surface CAR expression, cytotoxicity, and Th1 cytokine production, leading to reduced antitumor functionality. ${ }^{1} 2{ }^{3}$ To address these issues, studies have turned to genetically knocking out or overexpressing targets associated with an exhaustion or effector phenotype, such as PD-1 knockout (KO) and c-Jun overexpression, among other candidates that are typically receptors or transcription factors. ${ }^{4} 56$ However, there are other underexplored factors that mediate various aspects of immune regulation. While genome-wide CRISPR screens may discover such factors, they are unlikely to reveal phenotypes for genes whose function is partially redundant, therefore promising candidates may be missed. Such candidates include post-transcriptional regulators (PTRs) that coordinate immune responses, which are less well-studied in the context of CAR$\mathrm{T}$ cell function. We hypothesized that $\mathrm{KO}$ of these PTRs may increase CAR-T cell cytokine activity, phenotype, and persistence, potentially under long-term or exhaustion-inducing conditions, leading to increased tumor control. Ultimately, disruption of negative immune regulators could produce CAR$\mathrm{T}$ cells with enhanced activity and persistence, narrowing the gap between efficacy in hematological and solid tumors.

Methods To explore whether the disruption of two target PTRs improves solid tumor efficacy, we used CRISPR-Cas9 to genetically delete one or both PTRs in mesothelin-targeting human CAR-T cells and assayed their function in vitro and in vivo in NSG mice.
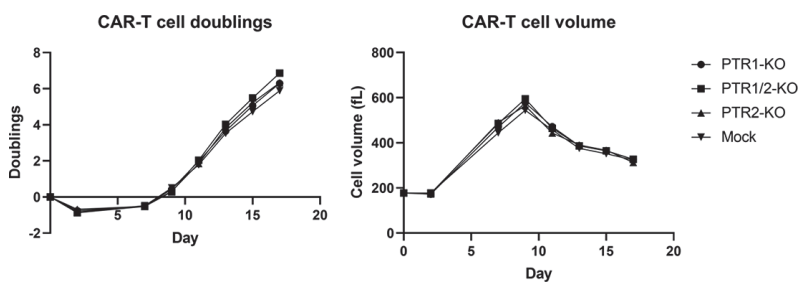

Abstract 131 Figure 1 Expansion kinetics of KO CAR-T cells

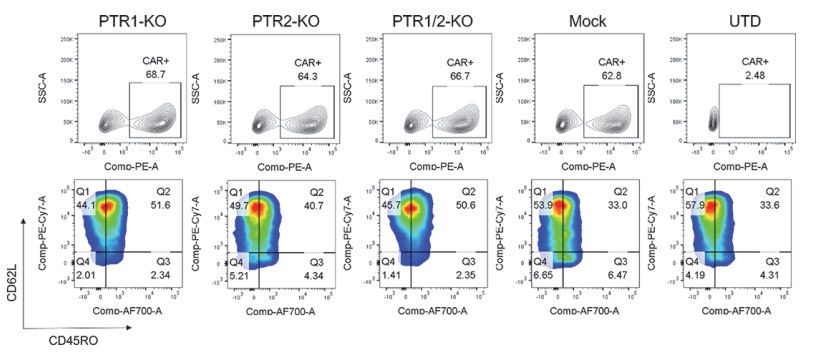

Abstract 131 Figure 2 Transduction efficiency and baseline phenotype of KO CAR-T cells

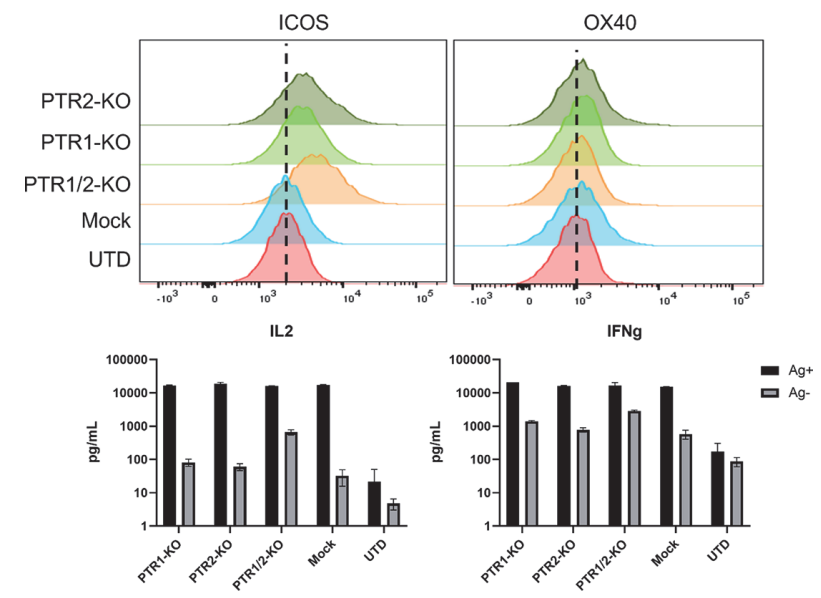

Abstract 131 Figure 3 Costimulatory receptor and cytokine expression of KO CAR-T cells

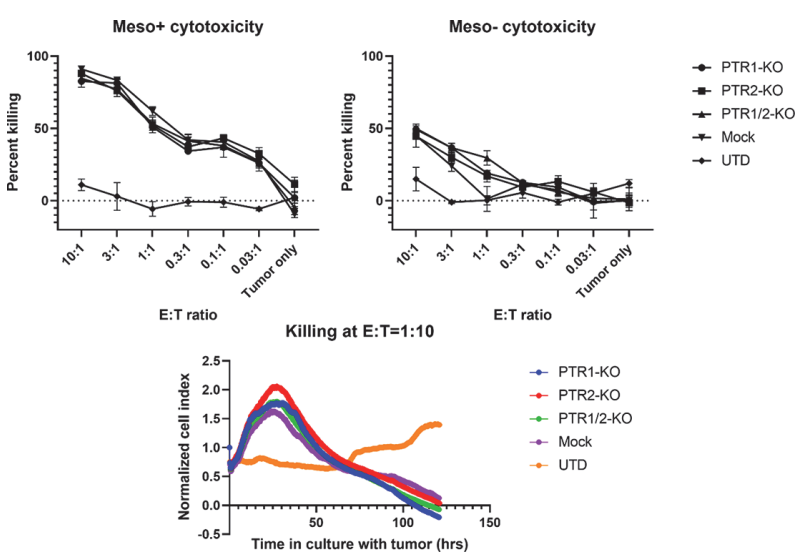

Abstract 131 Figure 4 In vitro cytotoxicity of KO CAR-T cells

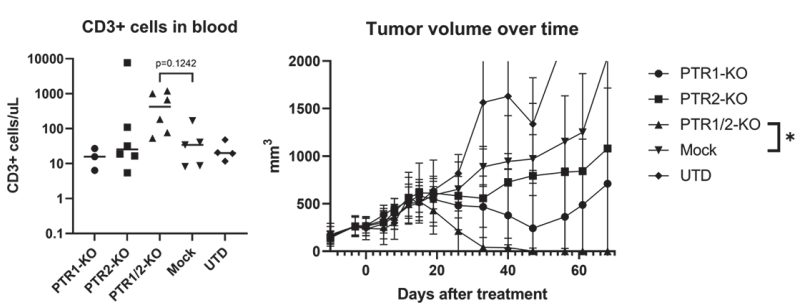

Abstract 131 Figure 5 In vivo activity of KO CAR-T cells

Results We show successful genetic deletion of these genes in post-thymic human $\mathrm{T}$ cells and that their disruption does not affect primary expansion (figure 1) or transduction efficiency (figure 2). These KO CAR-T cells display increased expression of co-stimulatory receptors and various cytokines (figure 3). While KO CAR-T cells are functionally similar to WT CAR-T cells in in vitro assays (figure 4), KO CAR-T cells demonstrate superior activity in vivo and can clear large, established tumors compared to WT CAR-T cells at low dose (figure 5). 
Conclusions These results indicate that $\mathrm{KO}$ of our target PTRs may improve the potency of CAR-T cells in solid tumors and may have important implications on the development of effective solid-tumor cell therapies.

\section{REFERENCES}

1. JE Wherry and M Kurachi, Molecular and cellular insights into T cell exhaustion, Nature Reviews Immunology 2015;15:486-499.

2. EW Weber, et al. Transient rest restores functionality in exhausted CAR-T cells through epigenetic remodeling. Science 2021;372:6537.

3. S Kuramitsu et al. Induction of T cell dysfunction and NK-like T cell differentiation in vitro and in patients after CAR T cell treatment. Cell, in revision.

4. BD Choi et al, CRISPR-Cas9 disruption of PD-1 enhances activity of university EGFRvIII CAR T cells in a preclinical model of human glioblastoma. Journal for ImmunoTherapy of Cancer 2019;7:304.

5. RC Lynn et al. c-Jun overexpression in CAR T cells induces exhaustion resistance. Nature 2019;576:293-300.

6. LJ Rupp et al. CRISPR/Cas9-mediated PD-1 disruption enhances anti-tumor efficacy of human chimeric antigen receptor T cells. Scientific Reports 2017;7:737.

http://dx.doi.org/10.1136/jitc-2021-SITC2021.131 